\title{
Supporting patients at home after colorectal surgery through a mobile app: A feasibility study
}

\author{
Christine Keng ${ }^{1,2}$, Selina Schmocker ${ }^{1}$, Saira Rashid ${ }^{1}$, Alifiya Goriawala ${ }^{1}$, Alexandra Easson ${ }^{1}$, Erin Kennedy ${ }^{1,2}$ \\ ${ }^{1}$ Department of Surgery, Mount Sinai Hospital, University of Toronto, Ontario, Canada \\ ${ }^{2}$ Institute of Health Policy and Management Evaluation, University of Toronto, Toronto, Ontario
}

\section{OBJECTIVE}

To assess feasibility of a mobile app for remote support and monitoring of patients after discharge home following colorectal surgery

Mobile app features daily patient questionnaire, tailored recommendations, education
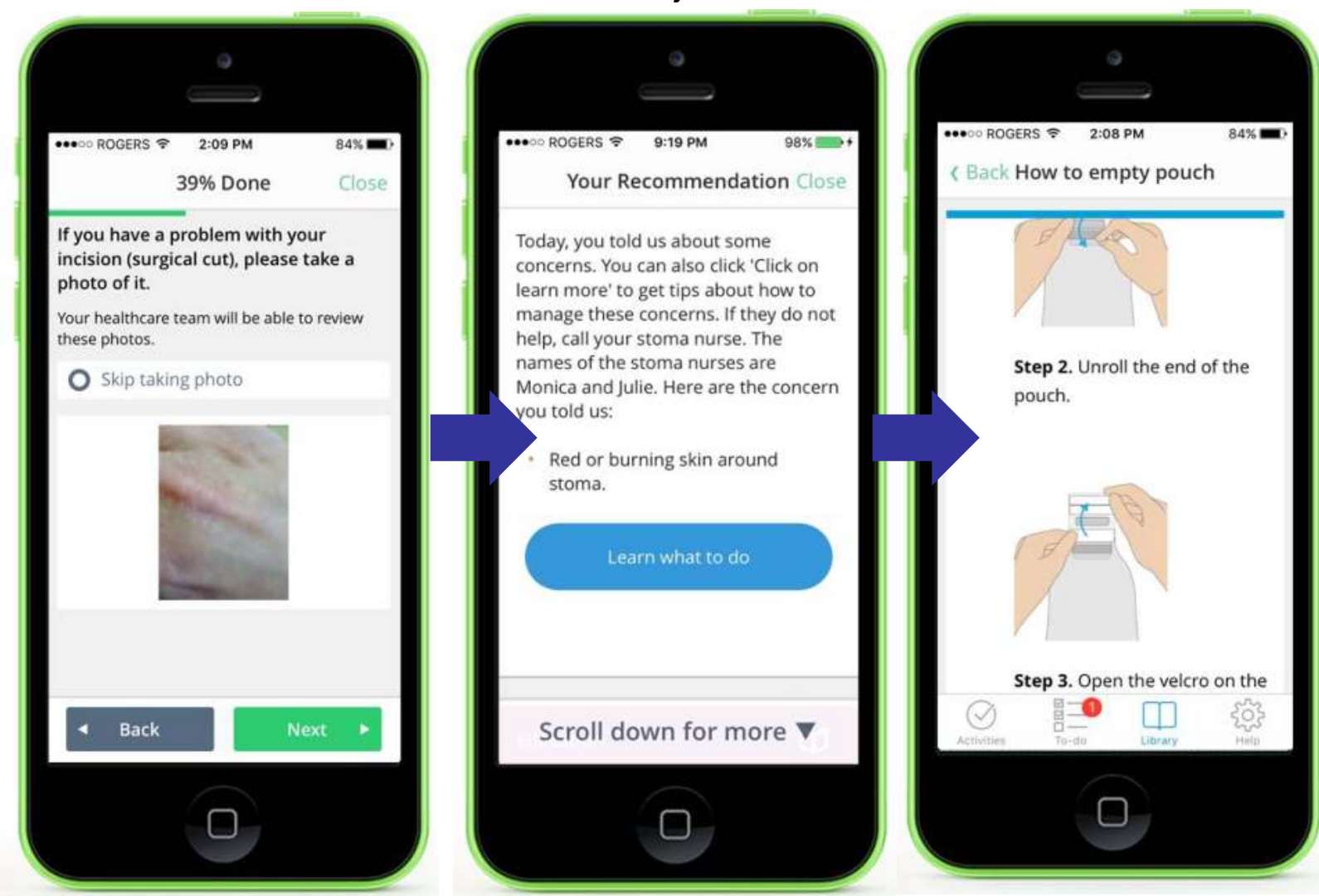

- Mobile app used over first 14 days after discharge

- Patients submitted daily symptom survey $\rightarrow$ received tailored feedback and education

- 2 Physician Assistants (PA) reviewed app entries daily

- 'Red flag' patient entries prompted call from PA or warning to patient to visit Emergency Room (ER)

\section{OUTCOMES}

- Feasibility outcomes

- App usage by patients

- Time spent by PAs monitoring app

- Patient satisfaction with app

- Exploratory outcomes

- ER visits within 30 days

- ER visits avoided

- 30-day readmissions to hospital

- Complications

Demographic and clinical characteristics of study sample $(n=81)$

\begin{tabular}{lc}
\hline \multicolumn{1}{c}{ Variable } & $\mathbf{n}(\%)$ \\
\hline Age & $43(33-55)$ \\
Female & $36(44.4)$ \\
Diagnosis & \\
$\quad$ Neoplasm/malignancy & $19(23.5)$ \\
Inflammatory bowel disease & $51(63.0)$ \\
Other & $11(13.6)$ \\
Surgical approach & \\
Laparoscopic & $38(46.9)$ \\
Open & $43(53.1)$ \\
Operation performed & \\
Partial/total colectomy & $33(40.7)$ \\
Low anterior resection & $8(9.9)$ \\
Abdominoperineal resection & $3(3.7)$ \\
Ileal pouch anal anastomosis & $10(12.3)$ \\
Other & $27(33.3)$ \\
Length of stay in days* & $6(4-8)$ \\
Stoma (ileostomy/colostomy) & $38(46.9)$ \\
\hline
\end{tabular}

* Expressed as median (range)

\section{RESULTS}

- Nov. 2016 to Apr. 2017, enrolled 106 patients who underwent colorectal surgery at Mount Sinai Hospital (Toronto, Ontario)

- 92 patients (86.8\%) logged in and used app at least once

- 81 patients with complete data and usage within first 14 days included in analysis

Mobile app usage and recommendations to patients

\begin{tabular}{lc}
\hline App usage and advice* & Mean (SD, range) \\
\hline App entries & $7.2(4.4,1-14)$ \\
Call backs & $2.0(1.9,0-9)$ \\
Warnings to visit ER & $0.2(0.6,0-4)$ \\
PA time (hours) monitoring app, per & 1.6 \\
week & \\
* Per patient, unless otherwise stated &
\end{tabular}

Mobile app usage in the first 14 days at home

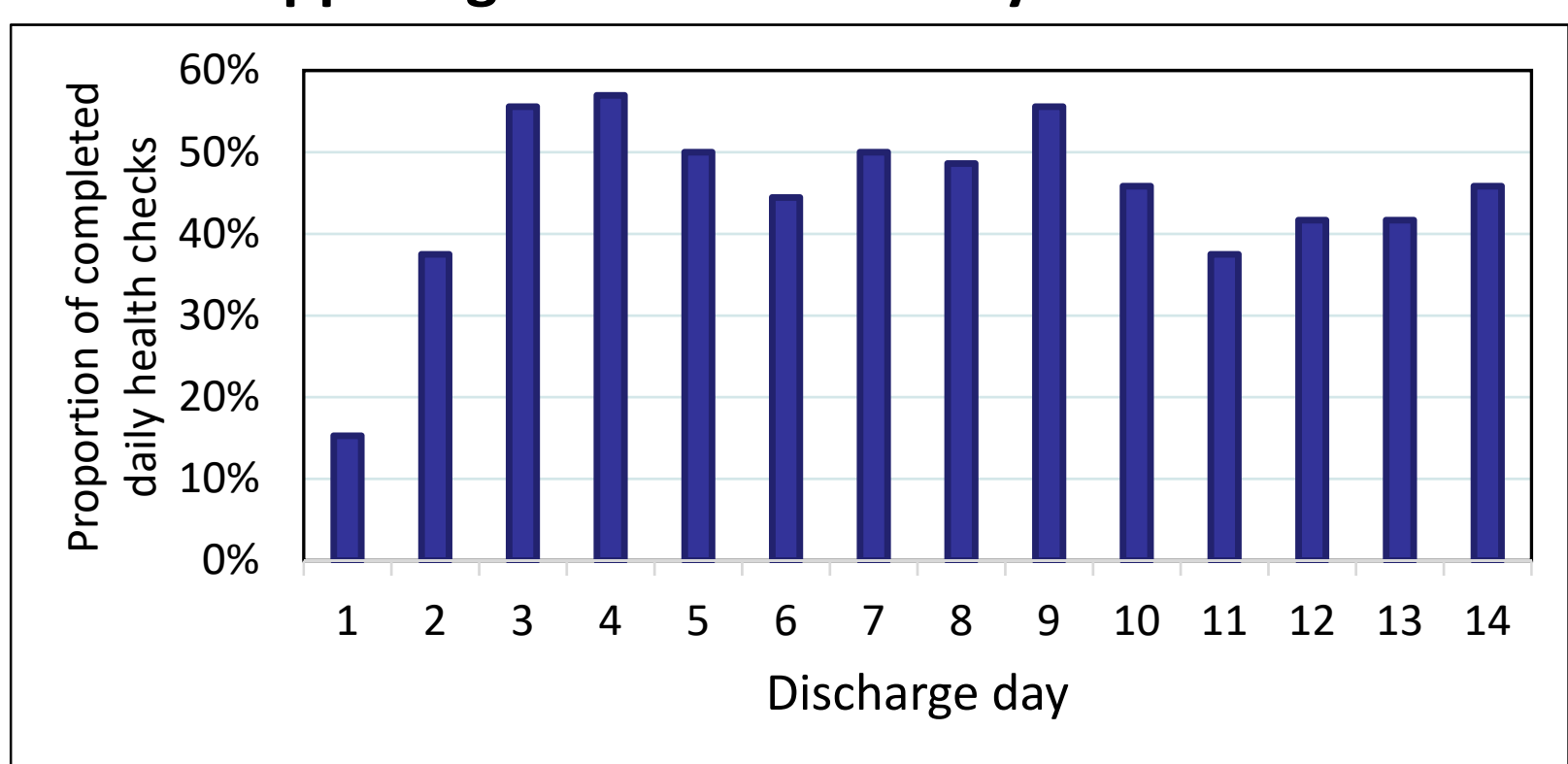

Patient satisfaction with mobile app:

- Surveys completed by $30.0 \%$ participants

- $100 \%$ reported overall experience as good or excellent

- $95.8 \%$ reported app helped them feel more confident in self-management at home

\section{Emergency room visits, readmissions, complications}

$(n=81)$

\begin{tabular}{lc}
\hline Outcome & $\mathbf{n}(\%)$ \\
\hline ER visits & $15(18.5)$ \\
ER visits avoided & $3(3.7)$ \\
Readmissions & $5(6.2)$ \\
Complications & \\
$\quad$ None & $47(58.0)$ \\
Ileus & $1(1.2)$ \\
Surgical site infection & $18(22.2)$ \\
Dehydration & $6(7.4)$ \\
Anastomotic leak & $1(1.2)$ \\
Obstruction & $2(2.5)$ \\
\hline
\end{tabular}

\section{CONCLUSION}

- A mobile app for patients post-colorectal surgery = feasible discharge intervention; viewed positively by patients

- Findings will inform future larger scale randomized studies to assess the effect of the mobile app on ER visits and readmissions among colorectal surgery patients. 